\title{
A Connection Formula for the $q$-Confluent Hypergeometric Function
}

Takeshi MORITA

Graduate School of Information Science and Technology, Osaka University, 1-1 Machikaneyama-machi, Toyonaka, 560-0043, Japan

E-mail:t-morita@cr.math.sci.osaka-u.ac.jp

Received October 09, 2012, in final form July 21, 2013; Published online July 26, 2013

http://dx.doi.org/10.3842/SIGMA.2013.050

Abstract. We show a connection formula for the $q$-confluent hypergeometric functions ${ }_{2} \varphi_{1}(a, b ; 0 ; q, x)$. Combining our connection formula with Zhang's connection formula for ${ }_{2} \varphi_{0}(a, b ;-; q, x)$, we obtain the connection formula for the $q$-confluent hypergeometric equation in the matrix form. Also we obtain the connection formula of Kummer's confluent hypergeometric functions by taking the limit $q \rightarrow 1^{-}$of our connection formula.

Key words: $q$-Borel-Laplace transformation; $q$-difference equation; connection problem; $q$ confluent hypergeometric function

2010 Mathematics Subject Classification: 33D15; 34M40; 39A13

\section{Introduction}

We show a new connection formula for two independent solutions to the $q$-confluent hypergeometric equation

$$
(1-a b q x) u\left(q^{2} x\right)-\{1-(a+b) q x\} u(q x)-q x u(x)=0 .
$$

We use notations in accordance with [2]. Assume that $q \in \mathbb{C}^{*}$ satisfies $0<|q|<1$ and $a / b$ is not an integer power of $q$. The basic hypergeometric series $r \varphi_{s}$ is defined by

$$
{ }_{r} \varphi_{s}\left(a_{1}, \ldots, a_{r} ; b_{1}, \ldots, b_{s} ; q, x\right):=\sum_{n \geq 0} \frac{\left(a_{1}, \ldots, a_{r} ; q\right)_{n}}{\left(b_{1}, \ldots, b_{s} ; q\right)_{n}(q ; q)_{n}}\left[(-1)^{n} q^{\frac{n(n-1)}{2}}\right]^{1+s-r} x^{n}
$$

where $(a ; q)_{n}$ is the $q$-shifted factorial

$$
\begin{aligned}
(a ; q)_{n} & := \begin{cases}1, & n=0 \\
(1-a)(1-a q) \cdots\left(1-a q^{n-1}\right), & n \geq 1,\end{cases} \\
(a ; q)_{\infty} & =\lim _{n \rightarrow \infty}(a ; q)_{n},
\end{aligned}
$$

and

$$
\left(a_{1}, a_{2}, \ldots, a_{m} ; q\right)_{\infty}=\left(a_{1} ; q\right)_{\infty}\left(a_{2} ; q\right)_{\infty} \cdots\left(a_{m} ; q\right)_{\infty}
$$

Equation (1) has solutions

$$
u_{1}(x)={ }_{2} \varphi_{0}(a, b ;-; q, x), \quad u_{2}(x)=\frac{(a b x ; q)_{\infty}}{\theta(-q x)}{ }_{2} \varphi_{1}\left(\frac{q}{a}, \frac{q}{b} ; 0 ; q, a b x\right)
$$


around the origin and has solutions

$$
v_{1}(x)=x^{-\alpha_{2} \varphi_{1}}\left(a, 0 ; \frac{a q}{b} ; q, \frac{q}{a b x}\right), \quad v_{2}(x)=x^{-\beta_{2} \varphi_{1}}\left(b, 0 ; \frac{b q}{a} ; q, \frac{q}{a b x}\right)
$$

around infinity. Here $q^{\alpha}=a$ and $q^{\beta}=b$.

The connection formula for a linear $q$-difference equation of the second order is a linear relation between $u_{1}(x), u_{2}(x)$ and $v_{1}(x), v_{2}(x)$ :

$$
\left(\begin{array}{l}
u_{1}(x) \\
u_{2}(x)
\end{array}\right)=\left(\begin{array}{ll}
C_{11}(x) & C_{12}(x) \\
C_{21}(x) & C_{22}(x)
\end{array}\right)\left(\begin{array}{l}
v_{1}(x) \\
v_{2}(x)
\end{array}\right)
$$

where the connection coefficients $C_{j k}(x)$ are $q$-periodic functions.

C. Zhang [10] proposed the connection formula for $u_{1}(x)$,

$$
\begin{aligned}
{ }_{2} f_{0}(a, b ; \lambda, q, x)= & \frac{(b ; q)_{\infty}}{\left(\frac{b}{a} ; q\right)_{\infty}} \frac{\theta(a \lambda)}{\theta(\lambda)} \frac{\theta\left(\frac{q a x}{\lambda}\right)}{\theta\left(\frac{q x}{\lambda}\right)} 2 \varphi_{1}\left(a, 0 ; \frac{a q}{b} ; q, \frac{q}{a b x}\right) \\
& +\frac{(a ; q)_{\infty}}{\left(\frac{a}{b} ; q\right)_{\infty}} \frac{\theta(b \lambda)}{\theta(\lambda)} \frac{\theta\left(\frac{q b x}{\lambda}\right)}{\theta\left(\frac{q x}{\lambda}\right)} 2 \varphi_{1}\left(b, 0 ; \frac{b q}{a} ; q, \frac{q}{a b x}\right),
\end{aligned}
$$

for $x \in \mathbb{C}^{*} \backslash[-\lambda ; q]$. Here ${ }_{2} f_{0}(a, b ; \lambda, q, x)$ is the $q$-Borel-Laplace transform of the divergent series ${ }_{2} \varphi_{0}(a, b ;-; q, x)$, i.e.,

$$
{ }_{2} f_{0}(a, b ; \lambda, q, x):=\mathcal{L}_{q, \lambda}^{+} \circ \mathcal{B}_{q}^{+} \varphi_{0}(a, b ;-; q, x) .
$$

In this paper, we show the following new connection formula for $u_{2}(x)$ :

$$
\begin{aligned}
{ }_{2} \varphi_{1}(q / a, q / b ; 0 ; q, a b x)= & \frac{(q / a ; q)_{\infty}}{(b / a ; q)_{\infty}} \frac{(a q x, 1 / a x ; q)_{\infty}}{(a b x ; q)_{\infty}}{ }_{2} \varphi_{1}(a, 0 ; a q / b ; q, 1 / a b x) \\
& +\frac{(q / b ; q)_{\infty}}{(a / b ; q)_{\infty}} \frac{(b q x, 1 / b x ; q)_{\infty}}{(a b x ; q)_{\infty}}{ }_{2} \varphi_{1}(b, 0 ; b q / a ; q, 1 / a b x) .
\end{aligned}
$$

Since $u_{1}(x)$ is a divergent series, the $q$-Stokes phenomenon appears in Zhang's connection formula. But our formula gives the exact relation between the convergent series $u_{2}(x)$ around the origin and the convergent series $v_{1}(x), v_{2}(x)$ around infinity.

The theta function of Jacobi is given by the series

$$
\theta_{q}(x):=\sum_{n \in \mathbb{Z}} q^{\frac{n(n-1)}{2}} x^{n}, \quad \forall x \in \mathbb{C}^{*},
$$

we denote $\theta(x)$ shortly. The theta function is written by the product form

$$
\theta(x)=\left(q,-x,-\frac{q}{x} ; q\right)_{\infty},
$$

which is known as Jacobi's triple product identity. For any $k \in \mathbb{Z}$, the theta function also satisfies the $q$-difference equation

$$
\theta\left(q^{k} x\right)=q^{-\frac{k(k-1)}{2}} x^{-k} \theta(x) .
$$

The theta function satisfies the inversion formula $\theta(x)=x \theta(1 / x)$. For all fixed $\lambda \in \mathbb{C}^{*}$, we define a $q$-spiral $[\lambda ; q]:=\lambda q^{\mathbb{Z}}=\left\{\lambda q^{k} ; k \in \mathbb{Z}\right\}$. Note that $\theta\left(\lambda q^{k} / x\right)=0$ if and only if $x \in[-\lambda ; q]$. 
At first, we review the confluent hypergeometric equation (CHGE). In 1813 [3], C.F. Gauss studied the hypergeometric series

$$
{ }_{2} F_{1}(\alpha, \beta ; \gamma ; z)=\sum_{n \geq 0} \frac{(\alpha)_{n}(\beta)_{n}}{(\gamma)_{n} n !} z^{n}, \quad \gamma \neq 0,-1,-2, \ldots,
$$

where $(\alpha)_{n}=\alpha\{\alpha+1\} \cdots\{\alpha+(n-1)\}$.

More generally, the generalized hypergeometric series is given by

$$
{ }_{r} F_{s}\left(\alpha_{1}, \ldots, \alpha_{r} ; \beta_{1}, \ldots, \beta_{s} ; z\right)=\sum_{n \geq 0} \frac{\left(\alpha_{1}\right)_{n} \cdots\left(\alpha_{r}\right)_{n}}{\left(\beta_{1}\right)_{n} \cdots\left(\beta_{s}\right)_{n} n !} z^{n}
$$

The hypergeometric function ${ }_{2} F_{1}(\alpha, \beta ; \gamma ; z)$ satisfies the second-order differential equation

$$
z(1-z) \frac{d^{2} u}{d z^{2}}+\{\gamma-(\alpha+\beta+1) z\} \frac{d u}{d z}-\alpha \beta u=0
$$

Gauss gave the connection formula for the function ${ }_{2} F_{1}(\alpha, \beta ; \gamma, z)$. We put $z \mapsto z / \beta$, take the limit $\beta \rightarrow \infty$ in equation (3), and obtain the confluent hypergeometric equation (CHGE)

$$
z \frac{d^{2} u}{d z^{2}}+(\gamma-z) \frac{d u}{d z}-\alpha u=0
$$

Solutions of (4) around the origin are

$$
\hat{u}_{1}(z)={ }_{1} F_{1}(\alpha ; \gamma ; z)
$$

and

$$
\hat{u}_{2}(z)=z^{1-\gamma}{ }_{1} F_{1}(\alpha-\gamma+1,2-\gamma, z) .
$$

Solutions around infinity are given by the divergent series

$$
\hat{v}_{1}(z)=(-z)^{-\alpha} F_{0}(\alpha, \alpha-\gamma+1 ;-; 1 / z)
$$

and

$$
\hat{v}_{2}(z)=e^{z} z^{\alpha-\gamma} F_{0}(1-\alpha, \gamma-\alpha ;-; 1 / z) .
$$

The asymptotic expansion of ${ }_{1} F_{1}(\alpha ; \gamma ; z)$ is given by

$$
\begin{aligned}
{ }_{1} F_{1}(\alpha ; \gamma ; z) \sim & \frac{\Gamma(\gamma)}{\Gamma(\gamma-\alpha)}(-z)^{-\alpha}{ }_{2} F_{0}(\alpha, \alpha-\gamma+1 ;-;-1 / z) \\
& +\frac{\Gamma(\gamma)}{\Gamma(\alpha)} e^{z} z^{\alpha-\gamma}{ }_{2} F_{0}(1-\alpha, \gamma-\alpha ;-; 1 / z),
\end{aligned}
$$

where $-\pi / 2<\arg z<3 \pi / 2$. Note that the connection formula for the second solution around infinity (5) can be derived from (6). In Section 2 we deal with another degeneration of equation (3) which is slightly different from the standard way.

It is known that there exists a $q$-analogue of ${ }_{2} F_{1}(\alpha, \beta ; \gamma ; z)$, which was introduced by E. Heine in 1847 as

$$
{ }_{2} \varphi_{1}(a, b ; c ; q, x):=\sum_{n \geq 0} \frac{(a ; q)_{n}(b ; q)_{n}}{(c ; q)_{n}(q ; q)_{n}} x^{n} .
$$


We assume that $c$ is not integer powers of $q$. The function ${ }_{2} \varphi_{1}(a, b ; c ; q, x)$ satisfies the secondorder $q$-difference equation $(q$-HGE)

$$
x(c-a b q x) \mathcal{D}_{q}^{2} u+\left[\frac{1-c}{1-q}+\frac{(1-a)(1-b)-(1-a b q)}{1-q} x\right] \mathcal{D}_{q} u-\frac{(1-a)(1-b)}{(1-q)^{2}} u=0,
$$

where $\mathcal{D}_{q}$ is the $q$-derivative operator defined for fixed $q$ by

$$
\mathcal{D}_{q} f(x)=\frac{f(x)-f(q x)}{(1-q) x} .
$$

By replacing $a, b, c$ by $q^{\alpha}, q^{\beta}, q^{\gamma}$ and then letting $q \rightarrow 1^{-}$, equation (7) tends to the hypergeometric equation (3). The $q$-hypergeometric equation (7) can be rewritten as

$$
(c-a b q x) u\left(q^{2} x\right)-\{c+q-(a+b) q x\} u(q x)+q(1-x) u(x)=0 .
$$

If we set $x \mapsto c x$ and $c \rightarrow \infty$ in (8), we obtain the $q$-confluent hypergeometric equation (1). Equation (1) is considered as a $q$-analogue of CHGE. Note that the first solution $u_{1}(x)$ is a divergent series and $u_{2}(x)$ is a convergent series around the origin. Therefore we should study the connection formula for $u_{1}(x)$ and $u_{2}(x)$ independently. We need different types of $q$-BorelLaplace transformations to obtain the connection formula for $u_{1}(x)$ and $u_{2}(x)$. This point is essentially different from the differential equation case.

We study connection problems for linear $q$-difference equations with irregular singular points. The irregularity of $q$-difference equations are studied using the Newton polygons by J.-P. Ramis, J. Sauloy and C. Zhang [6]. For any $q$-difference operator $\mathcal{P}=\sigma_{q}^{n}+a_{1}(z) \sigma_{q}^{n-1}+\cdots+a_{n}(z)$, the Newton polygon is defined as the convex hull of $\left\{(i, j) \in \mathbb{Z}^{2} \mid j \geq v_{0}\left(a_{i}\right)\right\}$, provided that $v_{0}$ are $z$-adic valuation in suitable fields. Graphically, the irregularity of $q$-difference equations and $q$-difference modules are the height of the Newton polygon (from the bottom to the upper right end).

Connection problems for linear $q$-difference equations [5] with regular singular points were studied by G.D. Birkhoff [1]. Linear $q$-difference equations have formal power series solutions $x^{\alpha} \sum_{n \geq 0} a_{n} x^{n}$ around the origin and $x^{\beta} \sum_{n \geq 0} b_{n} x^{-n}$ around infinity for generic exponents. But for connection problems for linear $q$-difference equations, we replace the function $x^{\kappa}$ with the function $\theta(x) / \theta(k x)$, where $k=q^{\kappa}$, since these functions satisfy the same $q$-difference equation $\sigma_{q} f(x)=q^{\kappa} f(x)$. Then, the fundamental system of solutions is given by single valued functions which have single poles at suitable $q$-spirals. Therefore, each connection coefficient has the periods $q$ and $e^{2 \pi i}$. The first example was given by G.N. Watson [7]. But a few examples of irregular singular cases are known $[4,8,9]$. In this paper, we give a connection formula for the $q$-confluent type function using the $q$-Borel-Laplace transformations.

In 1910, Watson [7] showed that the connection formula for the series ${ }_{2} \varphi_{1}(a, b ; c ; q, x)$ has the following form

$$
\begin{aligned}
{ }_{2} \varphi_{1}(a, b ; c ; q, x)= & \frac{(b, c / a ; q)_{\infty}}{(c, b / a ; q)_{\infty}} \frac{\theta(-a x)}{\theta(-x)} 2 \varphi_{1}\left(a, \frac{a q}{c} ; \frac{a q}{b} ; q, \frac{c q}{a b x}\right) \\
& +\frac{(a, c / b ; q)_{\infty}}{(c, a / b ; q)_{\infty}} \frac{\theta(-b x)}{\theta(-x)} 2 \varphi_{1}\left(b, \frac{b q}{c} ; \frac{b q}{a} ; q, \frac{c q}{a b x}\right) .
\end{aligned}
$$

Note that we can not set $a=0$ or $b=0$ directly in this formula.

In 2002, C. Zhang [10] showed one of the connection formula for the $q$-CHGE (2). The $q$-Borel-Laplace transformations were studied by C. Zhang in [10] (see Section 2 for more details). When we study connection problems for $q$-difference equations, this resummation 
method becomes a powerful tool. Note that we can find a new parameter $\lambda$ in the resummation ${ }_{2} f_{0}(a, b ; \lambda, q, x)$. Here $\lambda$ is the direction of the summation. This parameter brings us new viewpoints for the study of the $q$-Stokes phenomenon.

It is known that there exist two different types of the $q$-Borel-Laplace transformations.

The $q$-Borel-Laplace transformations of the first kind are defined in [10] and the $q$-BorelLaplace transformations of the second kind are studied in [9]. These $q$-Borel transformations are formal inverse transformations of each of the $q$-Laplace transformations.

C. Zhang presented a connection formula for the $\operatorname{series}{ }_{2} \varphi_{0}(a, b ;-; q, x)$ by the $q$-Borel-Laplace transformations of the first kind. But the connection formula for the second solution of (1) is not known. In this paper, we show the second connection formula for $q$-CHGE with the using of the $q$-Borel transformation and the $q$-Laplace transformation of the second kind. Combining with Zhang's connection formula, we obtain the connection formula in the matrix form (see Theorem 2). Using Watson's formula (9) we also give another proof of the new connection formula in Section 2.5.

In Section 3 we consider the limit $q \rightarrow 1^{-}$of our connection formula. If we take the limit $q \rightarrow 1^{-}$, we formally obtain the connection formula for the confluent hypergeometric series ${ }_{2} F_{0}$.

\section{The connection formula and the connection matrix}

We review a $q$-confluent hypergeometric equation in Section 2.1. Then we show a connection formula for the $q$-confluent hypergeometric function, which is different from Zhang's formula.

\subsection{Confluent hypergeometric equation}

For the confluent hypergeometric equation (3), we take another degeneration. We put $z \mapsto z \gamma$ and take the limit $\gamma \rightarrow \infty$. Then we obtain

$$
z^{2} \frac{d^{2} u}{d z^{2}}-\{1-(\alpha+\beta+1) z\} \frac{d u}{d z}+\alpha \beta u=0 .
$$

Solutions to (10) around the origin are given by the divergent series

$$
\tilde{u}_{1}(z)={ }_{2} F_{0}(\alpha, \beta ;-, z) \quad \text { and } \quad \tilde{u}_{2}(z)=e^{\frac{1}{z}}(-z)^{1-\alpha-\beta}{ }_{2} F_{0}(1-\alpha, 1-\beta ;-, z) .
$$

Solutions around infinity are given by the convergent series

$$
\tilde{v}_{1}(z)=(-z)^{\alpha}{ }_{1} F_{1}(\alpha, 1+\alpha-\beta,-1 / z) \quad \text { and } \quad \tilde{v}_{2}(z)=(-z)^{\beta}{ }_{1} F_{1}(\beta, 1+\beta-\alpha,-1 / z) .
$$

We consider a $q$-analogue of the confluent hypergeometric equation (8). The second-order $q$-difference equation

$$
\begin{gathered}
x\{a b q x-(1-q)\} D_{q}^{2} u(x)+\left\{1-\frac{(1-a)(1-b)-(1-a b q)}{1-q} x\right\} D_{q} u(x) \\
+\frac{(1-a)(1-b)}{(1-q)^{2}} u(x)=0 .
\end{gathered}
$$

can be rewritten as

$$
(1-a b q x) u\left(x q^{2}\right)-\{1-(a+b) q x\} u(x q)-q x u(x)=0,
$$

which is called a $q$-confluent hypergeometric equation. When we take $q \rightarrow 1^{-}$, the limit of (11) is the differential equation (8), provided that $a=q^{\alpha}, b=q^{\beta}$. 


\subsection{Local solutions to the $q$-confluent hypergeometric equation}

Consider the connection problem of (12). At first we show local solutions for (12) around $x=0$ and $x=\infty$.

Lemma 1. Equation (12) has solutions

$$
\begin{aligned}
& u_{1}(x)={ }_{2} \varphi_{0}(a, b ;-; q, x), \\
& u_{2}(x)=\frac{(a b x ; q)_{\infty}}{\theta(-q x)}{ }_{2} \varphi_{1}\left(\frac{q}{a}, \frac{q}{b} ; 0 ; q, a b x\right)
\end{aligned}
$$

around the origin and solutions

$$
\begin{aligned}
& v_{1}(x)=x^{-\alpha_{2} \varphi_{1}}\left(a, 0 ; \frac{a q}{b} ; q, \frac{q}{a b x}\right), \\
& v_{2}(x)=x^{-\beta} \varphi_{2}\left(b, 0 ; \frac{b q}{a} ; q, \frac{q}{a b x}\right),
\end{aligned}
$$

around inf inity, provided that $a=q^{\alpha}$ and $b=q^{\beta}$.

Proof. We show a fundamental system of solutions of (12) around $x=0$. If set $u(x)=\sum_{n \geq 0} a_{n} x^{n}$, $a_{0}=1$, then we obtain

$$
u_{1}(x)={ }_{2} \varphi_{0}(a, b ;-; q, x) .
$$

We set $\mathcal{E}(x)=1 / \theta(-q x)$ and $f(x)=\sum_{n>0} a_{n} x^{n}, a_{0}=1$ to obtain another solution solution around the origin. We assume that $u(x)=\overline{\mathcal{E}}(x) f(x)$. Note that the function $\mathcal{E}(x)$ has the following property

$$
\sigma_{q} \mathcal{E}(x)=-q x \mathcal{E}(x), \quad \sigma_{q}^{2} \mathcal{E}(x)=q^{3} x^{2} \mathcal{E}(x) .
$$

Therefore, we obtain the equation

$$
\left[q^{3} x(1-a b q x) \sigma_{q}^{2}+q\{1-(a+b) q x\} \sigma_{q}-q\right] f(x)=0 .
$$

Since the infinite product $(a b x ; q)_{\infty}$ satisfies the following $q$-difference relation

$$
\sigma_{q}\left[(a b x ; q)_{\infty}\right]=\frac{1}{1-a b x}(a b x ; q)_{\infty},
$$

we obtain the second solution. Therefore, solutions of equation (12) around the origin are given by

$$
u_{1}(x)={ }_{2} \varphi_{0}(a, b ;-; q, x), \quad u_{2}(x)=\frac{(a b x ; q)_{\infty}}{\theta(-q x)}{ }_{2} \varphi_{1}\left(\frac{q}{a}, \frac{q}{b} ; 0 ; q, a b x\right) .
$$

Around $x=\infty$, we can easily determine local solutions by setting

$$
v(x)=\frac{\theta(a \mu x)}{\theta(\mu x)} \sum_{n \geq 0} a_{n} x^{-n}, \quad a_{0}=1,
$$

for any fixed $\mu \in \mathbb{C}^{*}$ and $x \in \mathbb{C}^{*} \backslash[-\mu ; q]$.

Here $u_{1}(x)$ is a divergent series and $u_{2}(x), v_{1}(x)$ and $v_{2}(x)$ are convergent series [2]. Therefore, the $q$-Stokes phenomenon occurs for $u_{1}(x)$. 
Definition 1. For any $f(x)=\sum_{n \geq 0} a_{n} x^{n}$, the $q$-Borel transformation $\mathcal{B}_{q}^{+}$is

$$
\left(\mathcal{B}_{q}^{+} f\right)(\xi)=\varphi(\xi):=\sum_{n \geq 0} a_{n} q^{\frac{n(n-1)}{2}} \xi^{n}
$$

and the $q$-Laplace transformation $\mathcal{L}_{q, \lambda}^{+}$is

$$
\left(\mathcal{L}_{q, \lambda}^{+} \varphi\right)(x):=\sum_{n \in \mathbb{Z}} \frac{\varphi\left(q^{n} \lambda\right)}{\theta\left(\frac{q^{n} \lambda}{x}\right)} .
$$

C. Zhang determined a resummation of (13) by the $q$-Borel-Laplace transformations of the first kind as follows

$$
{ }_{2} f_{0}(a, b ; \lambda, q, x):=\mathcal{L}_{q, \lambda}^{+} \circ \mathcal{B}_{q}^{+}{ }_{2} \varphi_{0}(a, b ;-; q, x) .
$$

He also presented a connection formula (2) for ${ }_{2} f_{0}(a, b ; \lambda, q, x)$.

But the connection formula between (14) and (15), (16) is not known. In the next section, we show the second connection formula by means of the $q$-Borel-Laplace transformations of the second kind.

\subsection{The second connection formula}

We define the $q$-Borel transformation and the $q$-Laplace transformation of the second kind. These transformations are introduced by C. Zhang to obtain the solution of equation (17).

Definition 2. For $f(x)=\sum_{n \geq 0} a_{n} x^{n}$, the $q$-Borel transformation is defined by

$$
g(\xi)=\left(\mathcal{B}_{q}^{-} f\right)(\xi):=\sum_{n \geq 0} a_{n} q^{-\frac{n(n-1)}{2}} \xi^{n}
$$

and the $q$-Laplace transformation is

$$
\left(\mathcal{L}_{q}^{-} g\right)(x):=\frac{1}{2 \pi i} \int_{|\xi|=r} g(\xi) \theta_{q}\left(\frac{x}{\xi}\right) \frac{d \xi}{\xi} .
$$

Here $r>0$ is a sufficiently small number. The $q$-Borel transformation is considered as a formal inverse of the $q$-Laplace transformation.

Lemma 2 ([9]). We assume that the function $f$ can be q-Borel transformed to the analytic function $g(\xi)$ around $\xi=0$. Then, we have

$$
\mathcal{L}_{q}^{-} \circ \mathcal{B}_{q}^{-} f=f
$$

Proof. We can prove this lemma calculating residues of the $q$-Laplace transformation around the origin.

The $q$-Borel transformation satisfies the following operational relation.

Lemma 3. For any $l, m \in \mathbb{Z}_{\geq 0}$,

$$
\mathcal{B}_{q}^{-}\left(x^{m} \sigma_{q}^{l}\right)=q^{-\frac{m(m-1)}{2}} \xi^{m} \sigma_{q}^{l-m} \mathcal{B}_{q}^{-} .
$$


We apply the $q$-Borel transformation to equation (17) and use Lemma 3. We use the notation $g(\xi)$ as the $q$-Borel transform of $u_{2}(x)$. We check out that $g(\xi)$ satisfies the first-order $q$-difference equation

$$
g(q \xi)=\frac{(1+a q \xi)(1+b q \xi)}{\left(1+q^{2} \xi\right)} g(\xi) .
$$

Since $g(0)=a_{0}=1$, we have the infinite product of $g(\xi)$ as follows

$$
g(\xi)=\frac{\left(-q^{2} \xi ; q\right)_{\infty}}{(-q a \xi ; q)_{\infty}(-q b \xi ; q)_{\infty}} .
$$

Note that $g(\xi)$ has single poles at

$$
\left\{\xi \in \mathbb{C}^{*} ; \xi=-\frac{1}{a q^{k+1}},-\frac{1}{b q^{k+1}}, k \in \mathbb{Z}_{\geq 0}\right\} .
$$

We set

$$
r_{0}:=\max \left\{\frac{1}{|a q|}, \frac{1}{|b q|}\right\}
$$

and choose the radius $r>0$ such that $0<r<r_{0}$. By Cauchy's residue theorem, the $q$-Laplace transform of $g(\xi)$ is

$$
\begin{aligned}
f(x) & =\frac{1}{2 \pi i} \int_{|\xi|=r} g(\xi) \theta\left(\frac{x}{\xi}\right) \frac{d \xi}{\xi} \\
& =-\sum_{k \geq 0} \operatorname{Res}\left\{g(\xi) \theta\left(\frac{x}{\xi}\right) \frac{1}{\xi} ; \xi=-\frac{1}{a q^{k+1}}\right\}-\sum_{k \geq 0} \operatorname{Res}\left\{g(\xi) \theta\left(\frac{x}{\xi}\right) \frac{1}{\xi} ; \xi=-\frac{1}{b q^{k+1}}\right\},
\end{aligned}
$$

where $0<r<r_{0}$. Since there exists a positive constant $C_{N}$ (for any integer $N$ ) s.t.,

$$
|g(\xi)| \leq C_{N} \xi^{-N} .
$$

The following lemma plays a key role to calculate the residue.

Lemma 4. For any $k \in \mathbb{N}, \lambda \in \mathbb{C}^{*}$, we have:

1) $\operatorname{Res}\left\{\frac{1}{(\xi / \lambda ; q)_{\infty}} \frac{1}{\xi}: \xi=\lambda q^{-k}\right\}=\frac{(-1)^{k+1} q^{\frac{k(k+1)}{2}}}{(q ; q)_{k}(q ; q)_{\infty}}$,

2) $\frac{1}{\left(\lambda q^{-k} ; q\right)_{\infty}}=\frac{(-\lambda)^{-k} q^{\frac{k(k+1)}{2}}}{(\lambda ; q)_{\infty}(q / \lambda ; q)_{k}}, \quad \lambda \notin q^{\mathbb{Z}}$.

Summing up all residues, we obtain $f(x)$ as follows

$$
f(x)=\frac{\left(\frac{q}{a} ; q\right)_{\infty}}{\left(\frac{b}{a}, q ; q\right)_{\infty}} \frac{\theta(-a q x)}{\theta(-q x)}{ }_{2} \varphi_{1}\left(a, 0 ; \frac{a q}{b} ; q, \frac{q}{a b x}\right)+\frac{\left(\frac{q}{b} ; q\right)_{\infty}}{\left(\frac{a}{b}, q ; q\right)_{\infty}} \frac{\theta(-b q x)}{\theta(-q x)}{ }_{2} \varphi_{1}\left(b, 0 ; \frac{b q}{a} ; q, \frac{q}{a b x}\right) .
$$

Therefore, we obtain the following theorem.

Theorem 1. For any $x \notin[1 ; q]$, we have

$$
\begin{aligned}
u_{2}(x)= & \frac{(a b x ; q)_{\infty}}{\theta(-q x)} 2_{2} \varphi_{1}\left(\frac{q}{a}, \frac{q}{b} ; 0 ; q, a b x\right)=\frac{\left(\frac{q}{a} ; q\right)_{\infty}}{\left(\frac{b}{a}, q ; q\right)_{\infty}} \frac{\theta(-a q x)}{\theta(-q x)} 2 \varphi_{1}\left(a, 0 ; \frac{a q}{b} ; q, \frac{q}{a b x}\right) \\
& +\frac{\left(\frac{q}{b} ; q\right)_{\infty}}{\left(\frac{a}{b}, q ; q\right)_{\infty}} \frac{\theta(-b q x)}{\theta(-q x)} 2 \varphi_{1}\left(b, 0 ; \frac{b q}{a} ; q, \frac{q}{a b x}\right) .
\end{aligned}
$$




\subsection{The connection matrix}

Combining Zhang's connection formula and Theorem 1, we give the connection matrix for equation (1). At first, we define a new fundamental system of solutions around infinity. For any $\lambda$, $\mu \in \mathbb{C}^{*}, x \in \mathbb{C}^{*} \backslash[-\mu ; q], S_{\mu}(a, b ; q, x)$ is

$$
S_{\mu}(a, b ; q, x):=\frac{\theta(a \mu x)}{\theta(\mu x)} 2 \varphi_{1}\left(a, 0 ; \frac{a q}{b} ; q, \frac{q}{a b x}\right) .
$$

The function $\theta(a \mu x) / \theta(\mu x)$ satisfies the following $q$-difference equation

$$
u(q x)=\frac{1}{a} u(x),
$$

which is also satisfied by the function $u(x)=x^{-\alpha}, a=q^{\alpha}$. Note that the pair $\left(S_{\mu}(a, b ; q, x)\right.$, $\left.S_{\mu}(b, a ; q, x)\right)$ gives a fundamental system of solutions of equation (1) if $a / b \notin q^{\mathbb{Z}}$. We define $q$-elliptic functions $C_{\mu}^{\lambda}(a, b ; q, x)$ and $C_{\mu}(a, b ; q, x)$.

Definition 3. For any $\lambda, \mu \in \mathbb{C}^{*}$ we set functions $C_{\mu}^{\lambda}(a, b ; q, x)$ and $C_{\mu}(a, b ; q, x)$ as follows

$$
\begin{aligned}
C_{\mu}^{\lambda}(a, b ; q, x) & :=\frac{(b ; q)_{\infty}}{\left(\frac{b}{a} ; q\right)_{\infty}} \frac{\theta(a \lambda)}{\theta(\lambda)} \frac{\theta\left(\frac{q a x}{\lambda}\right)}{\theta\left(\frac{q x}{\lambda}\right)} \frac{\theta(\mu x)}{\theta(a \mu x)}, \\
C_{\mu}(a, b ; q, x) & :=\frac{\left(\frac{q}{a} ; q\right)_{\infty}}{\left(\frac{b}{a}, q ; q_{\infty}\right)} \frac{\theta(-a q x)}{\theta(-q x)} \frac{\theta(\mu x)}{\theta(a \mu x)} .
\end{aligned}
$$

Then $C_{\mu}^{\lambda}(a, b ; q, x)$ and $C_{\mu}(a, b ; q, x)$ are single valued as functions of $x$. They satisfy the following relation

$$
C_{\mu}^{\lambda}\left(a, b ; q, e^{2 \pi i} x\right)=C_{\mu}^{\lambda}(a, b ; q, x), \quad C_{\mu}^{\lambda}(a, b ; q, q x)=C_{\mu}^{\lambda}(a, b ; q, x)
$$

and

$$
C_{\mu}\left(a, b ; q, e^{2 \pi i} x\right)=C_{\mu}(a, b ; q, x), \quad C_{\mu}(a, b ; q, q x)=C_{\mu}(a, b ; q, x) .
$$

i.e. $C_{\mu}^{\lambda}(a, b ; q, x)$ and $C_{\mu}(a, b ; q, x)$ are $q$-elliptic functions. We set

$$
{ }_{2} f_{1}(a, b ; q, x):=u_{2}(x)=\frac{(a b x ; q)_{\infty}}{\theta(-q x)}{ }_{2} \varphi_{1}\left(\frac{q}{a}, \frac{q}{b} ; 0 ; q, a b x\right) .
$$

Thus, we obtain the connection formula in the matrix form.

Theorem 2. For any $\lambda, \mu \in \mathbb{C}^{*}, x \in \mathbb{C}^{*} \backslash[1 ; q] \cup[-\mu / a ; q] \cup[-\mu / b ; q] \cup[-\lambda ; q]$, we have

$$
\left(\begin{array}{c}
{ }_{2} f_{0}(a, b ; \lambda, q, x) \\
{ }_{2} f_{1}(a, b ; q, x)
\end{array}\right)=\left(\begin{array}{cc}
C_{\mu}^{\lambda}(a, b ; q, x) & C_{\mu}^{\lambda}(b, a ; q, x) \\
C_{\mu}(a, b ; q, x) & C_{\mu}(b, a ; q, x)
\end{array}\right)\left(\begin{array}{c}
S_{\mu}(a, b ; q, x) \\
S_{\mu}(b, a ; q, x)
\end{array}\right) .
$$

\subsection{Derivation from Watson's formula}

In this section, we give another proof of Theorem 1. Watson's formula (9) is a connection formula for the basic hypergeometric functions ${ }_{2} \varphi_{1}(a, b ; c ; q, x)$. We derive the connection formula

$$
\begin{aligned}
\frac{(a b x ; q)_{\infty}}{\theta(-q x)}{ }_{2} \varphi_{1}\left(\frac{q}{a}, \frac{q}{b} ; 0 ; q, a b x\right)= & \frac{(q / a ; q)_{\infty}}{(b / a, q ; q)_{\infty}} \frac{\theta(-a q x)}{\theta(-q x)} 2_{2} \varphi_{1}\left(a, 0 ; \frac{a q}{b} ; q, \frac{q}{a b x}\right) \\
& +\frac{(q / b ; q)_{\infty}}{(a / b, q ; q)_{\infty}} \frac{\theta(-b q x)}{\theta(-q x)} 2_{2} \varphi_{1}\left(b, 0 ; \frac{b q}{a} ; q, \frac{q}{a b x}\right)
\end{aligned}
$$

from Watson's formula (9). By take the limit $c \rightarrow 0$ of Watson's formula, we obtain the following proposition. 
Proposition 1 ([10]). For any $x \in \mathbb{C}^{*} \backslash q^{\mathbb{Z}}$, we have

$$
\begin{aligned}
{ }_{2} \varphi_{1}(a, b ; 0 ; q, x)= & \frac{(b ; q)_{\infty}}{(b / a ; q)_{\infty}} \frac{\theta(-a x)}{\theta(-x)} 1 \varphi_{1}\left(a ; \frac{a q}{b} ; q, \frac{q^{2}}{b x}\right) \\
& +\frac{(a ; q)_{\infty}}{(a / b ; q)_{\infty}} \frac{\theta(-b x)}{\theta(-x)} 1 \varphi_{1}\left(b ; \frac{b q}{a} ; q, \frac{q^{2}}{a x}\right) .
\end{aligned}
$$

In (19), we put $a \mapsto q / a, b \mapsto q / b$ and $x \mapsto a b x$, we obtain the relation as follows

Corollary 1. For any $x \in \mathbb{C}^{*} \backslash[a b ; q]$, we have

$$
\begin{aligned}
{ }_{2} \varphi_{1}\left(\frac{q}{a}, \frac{q}{b} ; 0 ; q, a b x\right)= & \frac{(q / b ; q)_{\infty}}{(a / b ; q)_{\infty}} \frac{\theta(-b q x)}{\theta(-a b x)}{ }_{1} \varphi_{1}\left(\frac{q}{a} ; \frac{b q}{a} ; q, \frac{q}{a x}\right) \\
& +\frac{(q / a ; q)_{\infty}}{(b / a ; q)_{\infty}} \frac{\theta(-a q x)}{\theta(-a b x)} 1 \varphi_{1}\left(\frac{q}{b} ; \frac{a q}{b} ; q, \frac{q}{b x}\right) .
\end{aligned}
$$

The function ${ }_{1} \varphi_{1}(a ; c ; q, x)$ is related to ${ }_{2} \varphi_{1}\left(a^{\prime}, 0 ; c^{\prime} ; q, x\right)$. Consider the relation between the function ${ }_{1} \varphi_{1}$ and the function ${ }_{2} \varphi_{1}$.

Proposition 2. For any $x \in \mathbb{C}^{*}$, we have

$$
{ }_{1} \varphi_{1}\left(a_{1} ; c_{1} ; q, \frac{c_{1} x}{a_{1}}\right)=(x ; q)_{\infty 2} \varphi_{1}\left(\frac{c_{1}}{a_{1}}, 0 ; c_{1} ; q, x\right)
$$

provided that $c_{1} / a_{1} \notin q^{\mathbb{Z}}$.

Proof. The function ${ }_{1} \varphi_{1}\left(a_{1} ; c_{1} ; q, c_{1} x / a_{1}\right)$ satisfies the equation

$$
\left[\left(c_{1}-c_{1} q x\right) \sigma_{q}^{2}-\left\{\left(c_{1}+q\right)-\frac{q c_{1}}{a_{1}} x\right\} \sigma_{q}+q\right] v(x)=0 .
$$

We set $v(x)=(x ; q)_{\infty} \tilde{v}(x)$, where $\tilde{v}(x):=\sum_{n \geq 0} \tilde{v}_{n} x^{n}$ and $\tilde{v}_{0}:=1$. Note that the function $(x ; q)_{\infty}$ satisfies the first-order $q$-difference equation

$$
\sigma_{q} f(x)=\frac{1}{1-x} f(x) .
$$

Then, we obtain the equation

$$
\left[\sigma_{q}^{2}-\left\{\left(1+\frac{q}{c_{1}}\right)-\frac{q x}{a_{1}}\right\} \sigma_{q}+\frac{q}{c_{1}}(1-x)\right] \tilde{v}(x)=0 .
$$

Equation (22) has the solution

$$
\tilde{v}(x)={ }_{2} \varphi_{1}\left(\frac{c_{1}}{a_{1}}, 0 ; c_{1} ; q, x\right) .
$$

Therefore, we obtain the conclusion.

Corollary 2. In (21), we put $a_{1} \mapsto q / a, c_{1} \mapsto b q / a$ and $x \mapsto q / a b x$. Then we obtain

$$
{ }_{1} \varphi_{1}\left(\frac{q}{a} ; \frac{b q}{a} ; q, \frac{q}{a x}\right)=\left(\frac{q}{a b x} ; q\right)_{\infty}{ }_{2} \varphi_{1}\left(b, 0 ; \frac{b q}{a} ; q, \frac{q}{a b x}\right) .
$$

We also put $a_{1} \mapsto q / b, c_{1} \mapsto a q / b$ and $x \mapsto q / a b x$. Then we obtain

$$
{ }_{1} \varphi_{1}\left(\frac{q}{b} ; \frac{a q}{b} ; q, \frac{q}{b x}\right)=\left(\frac{q}{a b x} ; q\right)_{\infty}{ }_{2} \varphi_{1}\left(a, 0 ; \frac{a q}{b} ; q, \frac{q}{a b x}\right) .
$$


By relations (20), (23) and (24),

$$
\begin{aligned}
\frac{(a b x ; q)_{\infty}}{\theta(-q x)}{ }_{2} \varphi_{1}\left(\frac{q}{a}, \frac{q}{b} ; 0 ; q, a b x\right) \\
=\frac{(q / b ; q)_{\infty}}{(a / b, q ; q)_{\infty}} \frac{\theta(-b q x)}{\theta(-a b x)} \frac{\left(q, a b x, \frac{q}{a b x} ; q\right)_{\infty}}{\theta(-q x)}{ }_{2} \varphi_{1}\left(b, 0 ; \frac{b q}{a} ; q, \frac{q}{a b x}\right) \\
\quad+\frac{(q / a ; q)_{\infty}}{(b / a, q ; q)_{\infty}} \frac{\theta(-a q x)}{\theta(-a b x)} \frac{\left(q, a b x, \frac{q}{a b x} ; q\right)_{\infty}}{\theta(-q x)}{ }_{2} \varphi_{1}\left(a, 0 ; \frac{a q}{b} ; q, \frac{q}{a b x}\right) \\
=\frac{(q / b ; q)_{\infty}}{(a / b, q ; q)_{\infty}} \frac{\theta(-b q x)}{\theta(-q x)} 2_{2} \varphi_{1}\left(b, 0 ; \frac{b q}{a} ; q, \frac{q}{a b x}\right) \\
\quad+\frac{(q / a ; q)_{\infty}}{(b / a, q ; q)_{\infty}} \frac{\theta(-a q x)}{\theta(-q x)} 2_{2} \varphi_{1}\left(a, 0 ; \frac{a q}{b} ; q, \frac{q}{a b x}\right) .
\end{aligned}
$$

Therefore, we obtain the formula (18).

\section{The limit $q \rightarrow 1^{-}$of the connection formula}

In this section we show the limit $q \rightarrow 1^{-}$of our connection formula. In [10], C. Zhang proposed the following limit.

Theorem 3 ([10]). For any $\alpha, \beta \in \mathbb{C}^{*}(\alpha-\beta \notin \mathbb{Z})$ and $z$ in any compact domain of $\mathbb{C}^{*} \backslash[-\infty, 0]$, we have

$$
\begin{aligned}
\lim _{q \rightarrow 1^{-}}{ }_{2} f_{0}\left(q^{\alpha}, q^{\beta} ; \lambda, q, \frac{z}{(1-q)}\right) \\
\quad=\frac{\Gamma(\beta-\alpha)}{\Gamma(\beta)} z^{-\alpha}{ }_{1} F_{1}\left(\alpha, \alpha-\beta+1 ; \frac{1}{z}\right)+\frac{\Gamma(\alpha-\beta)}{\Gamma(\alpha)} z^{-\beta}{ }_{1} F_{1}\left(\beta ; \beta-\alpha+1 ; \frac{1}{z}\right) .
\end{aligned}
$$

Our limit of the connection formula in Theorem 1 differs from the theorem above. By Theorem 1, we have

$$
u_{2}(x)=C_{\mu}(a, b ; q, x) S_{\mu}(a, b ; q, x)+C_{\mu}(b, a ; q, x) S_{\mu}(b, a ; q, x)
$$

for any $(x, q) \in \mathbb{C}^{*} \times(0,1]$.

The limit $q \rightarrow 1^{-}$of the left-hand side of $(25)$ is formally given by $e^{1 / z}(-z)^{1-\alpha-\beta}{ }_{2} F_{0}(1-$ $\alpha, 1-\beta ;-, z)$, provided that $a=q^{\alpha}, b=q^{\beta}$ and $x=z /(1-q)$. On the other hand, convergent series ${ }_{1} F_{1}(\alpha ; \alpha+1-\beta ; 1 / z)$ and ${ }_{1} F_{1}(\beta ; \beta+1-\alpha ; 1 / z)$ appear in the limit $q \rightarrow 1^{-}$of the right-hand side of $(25)$. The aim of this section is to prove the following theorem.

Theorem 4. The limit $q \rightarrow 1^{-}$of the new connection formula formally gives the following asymptotic formula

$$
\begin{aligned}
& e^{1 / z}(-z)^{1-\alpha-\beta}{ }_{2} F_{0}(1-\alpha, 1-\beta ;-, z)=\frac{\Gamma(\beta-\alpha)}{\Gamma(1-\alpha)}(-z)^{-\alpha}{ }_{1} F_{1}\left(\alpha ; \alpha+1-\beta ; \frac{1}{z}\right) \\
& +\frac{\Gamma(\alpha-\beta)}{\Gamma(1-\beta)}(-z)^{-\beta}{ }_{1} F_{1}\left(\beta ; \beta+1-\alpha ; \frac{1}{z}\right) .
\end{aligned}
$$

In [8], Zhang has shown a limit of theta functions, taking the principal value of the logarithm on $\mathbb{C}^{*} \backslash(-\infty, 0]$. 
Proposition 3. For any $\gamma \in \mathbb{C}^{*}$, we have

$$
\lim _{q \rightarrow 1^{-}} \frac{\theta\left(q^{\gamma} \frac{u}{1-q}\right)}{\theta\left(\frac{u}{1-q}\right)}(1-q)^{-\gamma}=u^{-\gamma}
$$

converges uniformly on compact subset of $\mathbb{C} \backslash(-\infty, 0]$.

We also remind the formulas for the $q$-gamma function $\Gamma_{q}(\cdot)$ and the $q$-exponential function $E_{q}(\cdot)$. The $q$-gamma function is defined by

$$
\Gamma_{q}(x):=\frac{(q ; q)_{\infty}}{\left(q^{x} ; q\right)_{\infty}}(1-q)^{1-x}, \quad 0<q<1
$$

This function satisfies $\lim _{q \rightarrow 1^{-}} \Gamma_{q}(x)=\Gamma(x)[2]$. The $q$-exponential function

$$
E_{q}(z)=\sum_{n \geq 0} \frac{q^{n(n-1) / 2}}{(q ; q)_{n}} z^{n}=(-z ; q)_{\infty}
$$

satisfies the limit

$$
\lim _{q \rightarrow 1^{-}} E_{q}(z(1-q))=e^{z}
$$

We set $a=q^{\alpha}, b=q^{\beta}$ and $x=z /(1-q)$ in Theorem 1 . We introduce the constant

$$
w(\alpha, \beta ; q):=(q ; q)_{\infty}(1-q)^{1-\alpha-\beta} .
$$

Consider the limit when $q \rightarrow 1^{-}$of each side of the identity of Theorem 1 . The limit of the left hand side of (18) is given by the following lemma.

Lemma 5. For any $\alpha, \beta \in \mathbb{C}^{*}, \alpha-\beta \notin \mathbb{Z}$, we have

$$
\begin{aligned}
\lim _{q \rightarrow 1^{-}} & w(\alpha, \beta ; q) \frac{\left(\frac{q^{\alpha+\beta} z}{1-q} ; q\right)}{\theta\left(-\frac{q z}{1-q}\right)} 2 \varphi_{1}\left(q^{1-\alpha}, q^{1-\beta} ; 0 ; q, \frac{q^{\alpha+\beta} z}{1-q}\right) \\
& =(-z)^{1-\alpha-\beta} e^{\frac{1}{z}}{ }_{2} F_{0}(1-\alpha, 1-\beta ;-, z) .
\end{aligned}
$$

Proof. Exploiting the fact

$$
\begin{aligned}
w(\alpha, \beta ; q) & \frac{\left(\frac{q^{\alpha+\beta} z}{1-q} ; q\right)_{\infty}}{\theta\left(-\frac{q z}{1-q}\right)} 2_{1}\left(q^{1-\alpha}, q^{1-\beta} ; 0 ; q, \frac{q^{\alpha+\beta} z}{1-q}\right) \\
= & \left\{\frac{\theta\left(q^{\alpha+\beta}\left(\frac{-z}{1-q}\right)\right)}{\theta\left(\frac{-z}{1-q}\right)}(1-q)^{-\alpha-\beta}\right\}\left\{\frac{\theta\left(\frac{-z}{1-q}\right)}{\theta\left(q\left(\frac{-z}{1-q}\right)\right)}(1-q)\right\} \\
& \times \frac{1}{E_{q}\left(-\frac{(1-q)}{q^{\alpha+\beta-1} z}\right)} 2_{1} \varphi_{1}\left(q^{1-\alpha}, q^{1-\beta} ; 0 ; q, \frac{q^{\alpha+\beta} z}{1-q}\right),
\end{aligned}
$$

we obtain the conclusion.

Consider the right-hand side of (18). 
Lemma 6. For any $\alpha, \beta \in \mathbb{C}^{*},(\alpha-\beta \notin \mathbb{Z})$, we have

$$
\begin{aligned}
\lim _{q \rightarrow 1^{-}} & w(\alpha, \beta, q)_{2} f_{1}\left(q^{\alpha}, q^{\beta} ; q, \frac{z}{(1-q)}\right) \\
\quad= & \frac{\Gamma(\beta-\alpha)}{\Gamma(1-\alpha)}(-z)^{-\alpha}{ }_{1} F_{1}\left(\alpha ; \alpha+1-\beta ; \frac{1}{z}\right)+\frac{\Gamma(\alpha-\beta)}{\Gamma(1-\beta)}(-z)^{-\beta}{ }_{1} F_{1}\left(\beta ; \beta+1-\alpha ; \frac{1}{z}\right) .
\end{aligned}
$$

Proof. Noting that

$$
\begin{aligned}
w(\alpha, \beta ; q) & \frac{\left(q^{1-\alpha} ; q\right)_{\infty}}{\left(q^{\beta-\alpha}, q ; q\right)_{\infty}} \frac{\theta\left(-\frac{q^{\alpha+1} z}{1-q}\right)}{\theta\left(-\frac{q z}{1-q}\right)}{ }_{2} \varphi_{1}\left(q^{\alpha}, 0 ; q^{\alpha+1-\beta} ; q, \frac{q^{1-\alpha-\beta}(1-q)}{z}\right) \\
= & \left\{\frac{\left(q^{1-\alpha} ; q\right)_{\infty}}{(q ; q)_{\infty}}(1-q)^{\alpha}\right\}\left\{\frac{(q ; q)_{\infty}}{\left(q^{\beta-\alpha} ; q\right)_{\infty}}(1-q)^{-(1-\beta+\alpha)}\right\} \\
& \times\left\{\frac{\theta\left(q^{\alpha+1}\left(\frac{-z}{1-q}\right)\right)}{\theta\left(\frac{-z}{1-q}\right)}(1-q)^{-\alpha-1}\right\}\left\{\frac{\theta\left(\frac{-z}{1-q}\right)}{\theta\left(q\left(\frac{-z}{1-q}\right)\right)}(1-q)\right\} \\
& \times{ }_{2} \varphi_{1}\left(q^{\alpha}, 0 ; q^{\alpha+1-\beta} ; q, \frac{q^{1-\alpha-\beta}(1-q)}{z}\right),
\end{aligned}
$$

we prove the lemma.

Finally, we obtain the proof of Theorem 4.

\section{Acknowledgements}

The author would like to give heartfelt thanks to Professor Yousuke Ohyama who provided carefully considered feedback and many valuable comments. The author also would like to thank the anonymous referees for their helpful comments.

\section{References}

[1] Birkhoff G.D., The generalized Riemann problem for linear differential equations and the allied problems for linear difference and q-difference equations, Proc. Amer. Acad. Arts Sci. 49 (1913), 521-568.

[2] Gasper G., Rahman M., Basic hypergeometric series, Encyclopedia of Mathematics and its Applications, Vol. 96, 2nd ed., Cambridge University Press, Cambridge, 2004.

[3] Gauss C.F., Disquisitiones generales circa seriem infinitam ..., in Werke, Bd. 3, Königlichen Gesellschaft der Wissenschaften zu Göttingen, 1866, 123-162.

[4] Morita T., A connection formula of the Hahn-Exton q-Bessel function, SIGMA 7 (2011), 115, 11 pages, arXiv:1105.1998.

[5] Ohyama Y., A unified approach to $q$-special functions of the Laplace type, arXiv:1103.5232.

[6] Ramis J.P., Sauloy J., Zhang C., Local analytic classification of $q$-difference equations, arXiv:0903.0853.

[7] Watson G.N., The continuation of functions defined by generalized hypergeometric series, Trans. Camb. Phil. Soc. 21 (1910), 281-299.

[8] Zhang C., Remarks on some basic hypergeometric series, in Theory and Applications of Special Functions, Dev. Math., Vol. 13, Springer, New York, 2005, 479-491.

[9] Zhang C., Sur les fonctions q-Bessel de Jackson, J. Approx. Theory 122 (2003), 208-223.

[10] Zhang C., Une sommation discrète pour des équations aux $q$-différences linéaires et à coefficients analytiques: théorie générale et exemples, in Differential Equations and the Stokes Phenomenon, World Sci. Publ., River Edge, NJ, 2002, 309-329. 